\title{
THE NEW RIEMANN-WEBER-VOLUME II
}

Die Differential- und Integralgleichungen der Mechanik und Physik. Zweiter, physikalischer Teil, edited by P. Frank with the assistance of H. Faxén, R. Fürth, Th. v. Kármán, Fr. Noether, C. W. Oseen, A. Sommerfeld, E. Trefftz. Braunschweig, Vieweg, 1927. 863 pp. +88 figures. Paper, 53 Rentenmark; bound, 58 Rentenmark.

The first part, or mathematical section of this work, was reviewed by the present writer in the May-June 1927 number of this Bulletin. It outlines in a very broad manner the mathematical tool-box of the theoretical physicist of the present day, which covers nearly the whole realm of analysis and large portions of the other departments of mathematics.

For the second volume, the goal adopted by the editors was to accomplish for the last Riemann-Weber volume of 1910, that which Weber did in 1900, for Riemann's original Partielle Differentialgleichungen der mathematischen Physik. We may quote freely from the preface to the effect that in spite of fad and fancy in physical hypotheses, the mathematical formulations and methods are relatively constant. Examples are cited in the application of the planetary perturbation theory of Laplace and Lagrange to atomic states of energy; in the identity between the equations for the transfer of heat due to Fourier and that of the phenomena of diffusion in liquids; in the universality of the wave equations of D'Alembert, Euler and Gauss in sound, light, and atomistics; in the appearance of classical potential theory of Laplace, Gauss, Cauchy and Riemann in the modern theories of lift and drag on an airplane wing. Contrarywise, the fluid theories of heat and electricity are in the discard, atoms, molecules and electrons may even follow, Newton's hypothesis of Universal Gravitation may go out of fashion, but the fundamental analytic framework in terms of which the corresponding phenomena are described is relatively permanent. More recently, the influence of the mathematical frame of reference on hypothesis has been considerable.

The second volume of the present work joins on to the first in very much the same manner as superstructure to foundation. Despite the fact that volume two is the work of seven writers, in each case different from each of the eight collaborators on the first, a unified picture of the entire field wherein differential and integral equations function in the field of mathematical physics has been secured. There is no discussion of merit or lack of merit of physical hypothesis, very little comparison between mathematical result and experimental observation, but for the sake of unity the entire purpose is concentrated on the development of those particular analytic formulations in the realm of physical phenomena, particularly to recent developments such as quanta, Brownian movements, radiation, etc., together with technical applications of special importance such as aeronautics and radio. Some of this material on these topics appears in print for the first time.

The following is a summary by chapter headings: 
Part I (by P. Frank). Analytic mechanics. 1. The differential equations of general mechanical systems. 2. Stability and small oscillations. 3 . The equations of motion of rigid bodies. 4. Methods in perturbation theory. 5. Problems of celestial and atomic mechanics.

Part II (by R. Fürth). Conduction of heat and diffusion. 6. Free conduction of heat and diffusion. 7. Induced conduction of heat and diffusion.

Part III (by Fr. Noether). The stationary and quasi-stationary electromagnetic fields. 8. Electrostatics. 9. Steady electric currents. 10. Magnetostatics. 11. Quasi-stationary currents and waves.

Part IV (by A. Sommerfeld). Electromagnetic oscillations. 12. General theorems and methods. 13. Theory of refraction. 14. Resistance and skin effect. 15. Waves on wires. 16. Wireless telegraphy.

Part V (by E. Trefftz). Mechanics of continua. 17. Mathematical foundations of the theory of elasticity. 18. Problems in elastic equilibrium. 19. Dynamical problems of elastic theory. 20. Ideal fluids (by Th. v. Kármán). 21. Motion of viscous fluids (by Faxén and Oseen).

Part $I$ is especially complete on the general equations of mechanics and their solution. The treatment is entirely classical and prepares the ground for the problems of classical atomic mechanics. Relativistic mechanics is not included. The general equations of motion and stability of an airplane are treated. Part II on heat and diffusion follows in the main the classical treatment, except that the problems of diffusion are treated in parallel with those of heat flow. Part III on the static and quasi-static electromagnetic fields covers both the classic and the modern portions of the subject. The same may be said of electromagnetic oscillations. Here, the emphasis is on the theory of radiation and electric wave phenomena with applications to radio-activity, light and wireless. Finally, Part IV treats the mechanics of continuous media such as elasticity, motion of fluids, both perfect and viscous, and related problems. The last two chapters of this section contain new material of importance in aerodynamics.

It is a fairly simple matter to point out omissions in the writing of as comprehensive a work as the present one. There is the immediate reply to this of the authors of a desire to avoid expansion into encyclopedic proportions. However, it does seem that it would have been in order to include some applications of difference equations or algebraic systems related to differential and integral equations. One might also venture to suggest the inclusion of some chapters on the newer geometry and the theory of geodesics with applications to problems in gravitation, elasticity, etc.

Though definitely outside of the program of the present two volumes, if one were to add in an appendix, chapters on statistics and probability in relation to states of energy, and group and number theory in relation to the concept of symmetries in crystal structure, one would have a truly representative and comprehensive picture of the interlacing of mathematical reference frames and physical hypotheses in the description of the natural phenomena of the universe.

H. J. EtTLINGER 